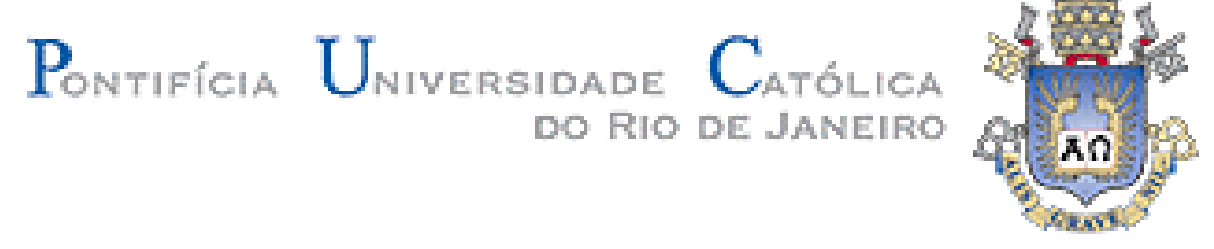

Fanny Herrera Loayza

Modelagem do Comportamento Pós-Sismo de uma

Barragem de Rejeito

Dissertação de Mestrado

Dissertação apresentada ao Programa de PósGraduação em Engenharia Civil da PUC-Rio como requisito parcial para obtenção do título de Mestre em Engenharia Civil.

Orientador: Prof. Celso Romanel

Rio de Janeiro

Março de 2009 
Fanny Herrera Loayza

\title{
Modelagem do Comportamento Pós-Sismo de uma Barragem de Rejeito
}

\begin{abstract}
Dissertação apresentada como requisito parcial para obtenção do grau de Mestre pelo Programa de PósGraduação em Engenharia Civil da PUC-Rio. Aprovada pela Comissão Examinadora abaixo assinada.
\end{abstract}

Prof. Celso Romanel

Orientador, PUC-Rio

Prof. Alberto S.F.J. Sayão PUC-Rio

Prof. João Luís Pascal Roehl

PUC-Rio

Francisco Claudio Pereira de Barros CNEN - Comissão Nacional de Energia Nuclear

Prof. José Eugênio Leal Coordenador Setorial do Centro

Técnico Científico - PUC-Rio

Rio de Janeiro, 31 de março de 2009 
Todos os direitos reservados. É proibida a reprodução total ou parcial do trabalho sem autorização da universidade, da autora e do orientador.

Fanny Herrera Loayza

Graduou-se em Engenharia Civil pela Pontifícia Universidade Católica do Peru - PUCP em 1996. Principais áreas de interesse: dinâmica de solos, geomecânica computacional e mineração

Ficha Catalográfica

Herrera Loayza, Fanny

Modelagem do comportamento pós-sismo de uma barragem de rejeito / Fanny Herrera Loayza ; orientador: Celso Romanel. - 2009.

$80 \mathrm{f}$ : : il.(color.) ; $30 \mathrm{~cm}$ 220 f. il; $29,7 \mathrm{~cm}$.

Dissertação (Mestrado em Engenharia Civil)Pontifícia Universidade Católica do Rio de Janeiro, Rio de Janeiro, 2009.

Inclui bibliografia

1. Engenharia civil - Teses. 2. Modelo numérico. 3. Comportamento pós-sismo. 4. Análise dinâmica. 5. Barragem de rejeito. 6. Mineração. I. Romanel, Celso. II. Pontifícia Universidade Católica do Rio de Janeiro. Departamento de Engenharia Civil. III. Título. 


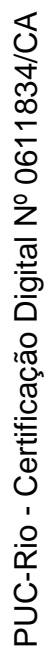

Ao amor da minha vida: meu amado esposo Fernando 


\section{Agradecimentos}

A Deus minha fonte de fé e esperança. Sem Ele nada seria possível!

Ao professor Celso Romanel, pela sua impecável orientação e dedicado acompanhamento do meu trabalho. Seu apoio, compreensão e paciência foram muito importantes para mim no desenvolvimento desta dissertação, que é produto do trabalho de ambos. Obrigada Mestre! Eu espero atender às suas expectativas, sua discípula promete tentar não desapontá-lo no futuro.

Ao meu pai, que desde o céu sempre me acompanha e cuida de mim. Sempre senti sua presença e sei que sem ele nunca teria alcançado meu ansiado sonho.

À minha mãe, minha "carinhosa". Você nunca me deixou sozinha. Obrigada pelas viagens tão sacrificadas para me visitar e que não as mereço. Sua companhia me fez sentir em casa no Brasil e sua alegria contagiante me ajudou nos momentos mais difíceis. Obrigada mãezinha, você é minha melhor amiga!

Ao meu irmão Pablo por seu apoio incondicional durante todo esse tempo. Obrigada por cuidar da mamãe na minha ausência e pelo incentivo para que eu fizesse o mestrado. À toda minha família, meus tios, primos e sobrinhos que sempre se preocuparam comigo e rezaram todos os dias para que eu cumprisse meu sonho.

À Maria Fernanda, Marianna, Paôla e Renata, companheiras do dia-a-dia na PUC.

À Adriana, Claudia, Dario, Martin e William, minha família no Brasil, pela grata convivência em casa e o apoio incondicional, em especial nos últimos meses do mestrado.

À Paôla pelo apoio na geração do sismo artificial para a aplicação do presente trabalho e pelas sugestões e recomendações para o melhor desenvolvimento da dissertação. 
Ao Jorge pelo apoio no conhecimento teórico, fornecimentos de artigos e, sobretudo, pela amizade.

À Vivian, minha grande amiga. Através de você conheci a generosidade do povo brasileiro para com os estrangeiros, por sua amizade e ajuda incondicional durante todo o mestrado.

À Cynthia, Sean, Tom, Graham, Mark, Mario e Olimpio, pelo fornecimento dos dados utilizados neste estudo e, além disso, pelo contínuo apoio e cooperação.

À professora Andréia Diniz de Almeida pelas sugestões em relação ao item de ameaça sísmica. Ao engenheiro Denys Parra pelo apoio inicial no fornecimento de dados de obras e pelas consultas técnicas. Ao professor Roehl pelo auxílio na elaboração do capítulo sobre aspectos de sismicidade. Ao professor Sayão pela ajuda na escolha dos parâmetros utilizados na barragem estudada neste trabalho e tradução de alguns termos técnicos. Ao professor Ramón Verdugo pelas respostas às minhas múltiplas dúvidas conceituais.

Aos meus amigos e colegas da PUC-Rio, pelo carinho e amizade.

Ao Departamento de Engenharia Civil da PUC-Rio pela infra-estrutura e suporte, e em especial a Rita, grande amiga.

À CAPES e à FAPERJ pelo apoio financeiro.

Finalmente, eu quero agradecer à pessoa a quem devo tudo, pelo seu apoio incondicional em todo este tempo e pela sua compreensão. Obrigada pelas várias ligações diárias e pelas oito viagens ao Brasil para me visitar. Eu te agradeço pelos momentos inesquecíveis e adoráveis que vivemos juntos no Brasil e no Peru. Eu dou graças a Deus todos os dias por ter me enviado você, e espero ansiosamente começar uma nova etapa da minha vida a seu lado. Meu amado esposo Fernando, meu amor, eu te peço perdão por todo este tempo que te deixei sozinho. A única coisa que eu posso dizer agora é que eu TE AMO MUITO. 


\section{Resumo}

Loayza Fanny Herrera; Romanel, Celso (orientador) Modelagem do Comportamento Pós-Sismo de uma Barragem de Rejeito. Rio de Janeiro, 2009. 220 p. Dissertação de Mestrado - Departamento de Engenharia Civil, Pontifícia Universidade Católica do Rio de Janeiro.

Os danos causados em geo-estruturas devido à ação de carregamentos dinâmicos gerados por terremotos não ocorrem necessariamente durante o evento, tendo sido observado em campo que muitos acontecem após o término da excitação. Esta dissertação tem como objetivo apresentar um fluxo de trabalho que compreenda o estado da prática da avaliação completa de uma barragem composta pelas análises estática, dinâmica e pós-sísmica. Para desenvolver a metodologia de trabalho proposta, utilizou-se o programa FLAC, software comercial de maior uso em análises dinâmicas detalhadas. A simulação neste programa de diferenças finitas permitiu o aprendizado de suas potencialidades, a aplicação de técnicas de modelagem e o conhecimento de suas limitações. A estrutura destinada à aplicação foi uma barragem de contenção de rejeitos de mineração localizada na Argentina. Os aspectos investigados compreendem a análise estática e a determinação do estado permanente, que marca o estado incial da etapa sísmica, a avaliação dinâmica e a análise de estabilidade pós-sismo. $\mathrm{Na}$ avaliação dinâmica no FLAC foram estudadas as condições de contorno mais adequadas aos requerimentos do problema. Também abrangeu-se a determinação do tipo de amortecimento e seus respetivos parâmetros. Finalmente, estimou-se a história no tempo de tensões, das velocidades e dos deslocamentos que compõem o estado final logo ao término do sismo, com o qual foi efetuada a análise póssismo. Analisou-se então a estabilidade do talude no mesmo programa. Em paralelo, também foram executados procedimentos alternativos para a avaliação pós-sismo, demonstrando, para a estrutura estudada, a importância e a praticidade da modelagem completa no FLAC.

\section{Palavras - chave}

Modelo numérico; comportamento pós-sismo; análise dinâmica; barragem de rejeito; mineração. 


\section{Abstract}

Loayza Fanny Herrera. Romanel, Celso (advisor). Modeling the PostSeismic Behavior of a Tailing Dam. Rio de Janeiro, 2009. 220 p. M.Sc. Dissertation - Departamento de Engenharia Civil, Pontifícia Universidade Católica do Rio de Janeiro.

The damages caused in geo-structures due to the action of dynamic loads produced by earthquakes do not take place necessarily during the event, being observed in the field that many of them happen after the end of the excitation. Among the factors that contribute to this behavior can be mentioned the generation and subsequent redistribution of porepressures, the action of erosive phenomena (piping) in cracks created by the seism and, mainly, the loss of the shear strength of the soil. For geo-structures located in zones of seismic activity, the consideration of post-seismic analyses, such as the stability of dam slopes, is a very important design requirement As for the estimate and distribution of the porepressure values generated by the earthquake, a computational simulation of the event is necessary, including the consideration of constitutive relations formulated in terms of effective stresses to well represent the occurrence of shear and volumetric strains of the soil and the possibility of the development of dynamic liquefaction. In this thesis, which investigates the post-seismic behavior of a tailing dam situated in Argentina, the numerical simulation of the dynamic response of the structure has been obtained using the computational code FLAC v.5, one of the most complete software nowadays available for analyses of geotechnical problems. A description of the numerical procedures, the difficulties, advantages and limitations in the use of FLAC will be discussed along this work.

\section{Keywords}

Numerical model; post-seismic behavior; dynamic analysis; tailing dams; mining 


\section{Sumário}

1 Introdução 29

1.1. Motivação e objetivos 29

1.2. Estrutura da dissertação 32

2 Fundamentos de sismicidade $\quad 34$

2.1. Conceitos gerais 34

2.1.1. Estrutura da Terra 34

2.1.2. Ondas planas de tensão 35

2.1.3. Falhas geológicas $\quad 39$

2.2. Origem dos sismos 41

2.2.1. Tectônica de placas 41

2.3. Teoria da recuperação elástica (elastic rebound theory) 44

2.4. Localização de um sismo 44

2.5. Grandeza de um sismo 46

2.5.1. Intensidade 46

2.5.2. Magnitude 46

2.6. Parâmetros do movimento do terreno 48

2.6.1. Parâmetros de amplitude $\quad 49$

2.6.2. Parâmetros de conteúdo de frequências 49

2.6.3. Parâmetros de duração 50

2.7. Estimativa dos parâmetros do movimento 51

2.7.1. Desenvolvimento das relações de prognóstico 51

2.7.2. Estimativa dos parâmetros de amplitude 52

2.7.3. Estimativa dos parâmetros de conteúdo de frequências 53

2.7.4. Estimativa da duração $\quad 54$

2.8. Projeto do movimento do terreno 54

2.8.1. Efeitos das condições do sítio no movimento do terreno 54

2.8.2. Parâmetros do projeto 55

2.8.3. Geração de movimento artificial do terreno 56 
2.8.4. Geração de sismos artificiais no domínio da frequência

2.9. Avaliação de ameaça sísmica 63

2.9.1. Análise determinística 63

2.9.2. Análise probabilística 65

3 Análise Pós-Sismo 70

3.1. Conceitos fundamentais e terminologia usada 71

3.1.1. Estado permanente 71

3.1.2. O fenômeno de liquefação 71

3.1.3. Susceptibilidade à liquefação 72

3.1.4. Parâmetro de estado 73

3.1.5. Potencial de liquefação 73

3.1.6. Razão de tensão cíclica e razão de resistência cíclica 74

3.1.7. Resistência cisalhante não drenada residual 75

3.1.8. Curva base para areia limpa do SPT (SPT clean-sand base curve)75

3.2. Determinação da CRR por meio de ensaios de campo 76

3.2.1. Ensaios de penetração padrão (standard penetration test, SPT) 76

3.2.2. Ensaio de cone (Cone penetration test, CPT) 79

3.2.3. Ensaios geofísicos para determinar a velocidade da onda cisalhante, $v_{s} \quad 83$

3.2.4. Ensaios de penetração Becker (Becker penetration test, BPT) 85

3.2.5. Fatores de correção 86

3.3. Cálculo da resistência ao cisalhamento não drenada 87

3.3.1. Cálculo da resistência ao cisalhamento não-drenada na condição permanente (Poulos et al.,1985) 88

3.3.2. Cálculo da resistência ao cisalhamento não drenada residual (Seed

\& Harder, 1990) 91

3.3.3. Cálculo da resistência não drenada crítica (Stark \& Mesri, 1992) 92

3.3.4. Cálculo da resistência à liquefação (Olson \& Stark, 2002) 94

3.4. Procedimento de análise de estabilidade pós-sismo 98

3.4.1. Estimar o potencial de liquefação 99

3.4.2. Estimativa da resistência ao cisalhamento não drenada reduzida 99

$\begin{array}{ll}\text { 3.4.3. Análise de estabilidade } & 101\end{array}$ 
4 Modelos Constitutivos 103

4.1. Modelo elástico 104

4.2. Modelo de Mohr Coulomb 106

$\begin{array}{ll}\text { 4.3. Modelo de Finn } & 108\end{array}$

4.3.1. Procedimento para avaliar o incremento da poropressão (Martin et $\begin{array}{ll}\text { al., 1975). } & 109\end{array}$

4.3.2. Método simplificado para calcular a geração e dissipação de poropressões (Seed et al., 1975). 115

4.3.3. Modelo de tensões efetivas para liquefação (Finn et al., 1977). 121

4.4. Modelo de Byrne 126

4.5. Modelo UBCsand 133

4.5.1. Comportamento elástico 135

4.5.2. Comportamento plástico mobilizado no plano de tensão de cisalhamento máximo 135

4.5.3. Comportamento plástico mobilizado no plano horizontal 137

4.6. Observações 140

5 Modelagem Numérica de Barragens de Terra 141

5.1. Características gerais do programa FLAC 142

5.2. Mecanismo inicial da modelagem da barragem 143

5.2.1. Configuração do projeto 143

5.2.2. Geração de malha 144

5.3. Modelagem estática 146

5.3.1. Modelos constitutivos 146

$\begin{array}{ll}\text { 5.3.2. Propriedades dos materiais } & 147\end{array}$

5.3.3. Interação fluido-mecânica 148

5.3.4. Condições iniciais e de contorno 149

5.4. Modelagem dinâmica 150

5.4.1. Processamento do registro da aceleração 151

5.4.2. Discretização da malha para a transmissão de ondas 153

5.4.3. Amortecimento mecânico 153

5.4.4. Aferição com o programa SHAKE 159 
5.4.5. Condições de contorno dinâmicas 161

5.5. Fator de segurança 164

5.6. Limitações do FLAC 165

5.6.1. Simulação em pequenas e grandes deformações 165

$\begin{array}{ll}\text { 5.6.2. Uso do amortecimento mecânico } & 167\end{array}$

5.6.3. Unificação dos tempos de processamento 168

5.6.4. Uso limitado do fator de segurança 168

$\begin{array}{ll}\text { 5.7. Observações } & 169\end{array}$

6 Comportamento Dinâmico e Pós-Sísmico de uma Barragem na Argentina

6.1. Descrição geral da estrutura 170

6.2. Propriedades do material 173

6.3. Configuração preliminar 174

6.3.1. Discretização da malha 175

6.3.2. Determinação das condições iniciais e de contorno estáticas 176

6.4. Simulação estática 178

6.4.1. Síntese dos resultados da análise estática 178

6.4.2. Determinação do fator de segurança estático 181

6.4.3. Determinação do fator de segurança pseudo-estático 182

6.5. Simulação dinâmica 183

$\begin{array}{ll}\text { 6.5.1. Sismicidade } & 184\end{array}$

6.5.2. Geração do sismo artificial 184

6.5.3. Processamento do registro sísmico 186

6.5.4. Frequência predominante da barragem 189

6.5.5. Aferição com o programa SHAKE 189

6.5.6. Síntese dos resultados da análise dinâmica 193

6.6. Simulação pós-sismo 199

6.6.1. Determinação do fator de segurança 200

6.6.2. Comparação com outros métodos de avaliação da análise de estabilidade pós-sismo 202

$\begin{array}{ll}\text { 6.7. Observações } & 204\end{array}$

7 Conclusões e Sugestões 206 
7.1. Conclusões 206

7.2. Sugestões 210

Referências Bibliográficas $\quad 212$

$\begin{array}{ll}\text { Apêndice } & 219\end{array}$

$\begin{array}{ll}\text { Anexo } & 220\end{array}$

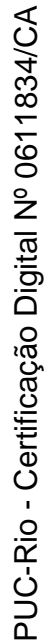




\section{Lista de figuras}

Figura 1.1 - Deslizamento na barragem de San Fernando, em 1971

(EERC, University of California, Berkeley, USA).

Figura 1.2 - Ruptura e reconstrução das condições iniciais da

barragem de San Fernando. Modificado de Seed et. al. (1988)

Figura 2.1- Esquema da estrutura da Terra

(www.ige.unicamp.br/site/aulas/109/Terra-tempo_geo-aula1.pdf).

Figura 2.2 - Movimentos de partícula produzidos pelos diferentes

tipos de ondas planas de tensão (Teixeira et al., 2003).

Figura 2.3 - Ondas sísmicas registradas a $10.000 \mathrm{~km}$ do epicentro:

a) sismo de foco profundo; b) sismo de foco superficial.

Modificado de Sauter (1989) apud Arias (1996).

Figura 2.4 - Notação geométrica para descrição do plano de falha

Figura 2.5 - Placas tectônicas principais

(http://pubs.usgs.gov/gip/dynamic/slabs.html).

Figura 2.6 - Movimentos interplacas

Figura 2.7 - Sismos ocorridos no Brasil da época colonial

ao ano 2000 (Berrocal, 1984).

Figura 2.8 - Elementos para descrição da localização de um sismo

Figura 2.9 - Representação de um espectro de resposta

com quatro escalas logarítmicas (adaptado de Figuereido, 2004).

Figura 2.10 - Geração artificial de movimentos de terreno

(adaptado de Kramer, 1996).

Figura 2.11 - Exemplo de uma função sintética no tempo gerada no

domínio da frequência (modificado de Kramer, 1996).

Figura 2.12 - Função Intensidade para um sismo com duração total de 15s (Figueiredo, 2004).

Figura 2.13 - Diagrama de blocos que descreve o procedimento de geração de sismos artificiais (Notas de aula de Dinâmica de Solos, 1996).

Figura 2.14 - Procedimento de avaliação da ameaça sísmica

determinística (modificado de Kramer, 1996). 
Figura 2.15 - Esquema de avaliação da ameaça sísmica

probabilística (NAHB Research Center, 2003).

Figura 3.1 - Curva base para areia limpa do SPT para sismo de magnitude 7,5 obtidos com dados de casos históricos.

Modificado de Seed et al. (1985) apud Youd et al. (2001).

Figura 3.2 - Curva recomendada para o cálculo de CRR baseada em

dados de liquefação empírica obtidos com CPT compilados de casos

históricos. Adaptado de Robertson \& Wride (1998) apud Youd et al. (2001). 80

Figura 3.3 - Gráfico que classifica o tipo de comportamento do solo

baseado no CPT. Modificado de Robertson (1990) apud Youd et al. (2001).

Figura 3.4 - Relação de liquefação recomendada para solos

limpos não cimentados baseados em dados de liquefação compilados

de casos históricos.

Adaptado de Andrus \& Stokoe (2000) apud Youd et al. (2001).

Figura 3.5 - Correlação entre a resistência à penetração Becker corrigida

$N_{B C}$ e a resistência do SPT corrigida $N_{60}$.

Modificado de Harder \& Seed (1986) e Harder (1997) apud Youd et al. (2001). 86

Figura 3.6 - Correção da resistência não drenada no estado permanente

medida para diferenças entre o índice de vazios in-situ e o índice de

vazios de laboratório (adaptado de Poulos et al., 1985).

Figura 3.7 - Relação entre a contagem de golpes corrigidos

para areia limpa $\left(N_{l}\right)_{60-C S}$ e a resistência não drenada residual $\left(S_{r}\right)$

baseadas em casos históricos (modificado de Seed \& Harder, 1990).

Figura 3.8 - Relação entre a razão da resistência não drenada crítica

e a contagem do número de golpes para uma areia limpa equivalente

(adaptado de Stark \& Mesri, 1992).

Figura 3.9 - Diagrama de corpo livre utilizado para a análise cinética

(modificado de Olson \& Stark, 2002).

Figura 3.10 - Relação da razão da resistência do solo liquefeito

baseada na resistência de ponta normalizada do CPT

(adaptado de Olson \& Stark, 2002).

Figura 3.11 - Determinação do potencial de liquefação

(modificado de Kramer, 1996). 
Figura 3.12 - Relações típicas entre a razão de excesso de poropressão residual e o fator de segurança contra liquefação para areia e para cascalho obtidos com dados de laboratório (adaptado de Marcuson et al., 1990).

Figura 4.1 - Critério de escoamento de Mohr-Coulomb:

a) no plano $(\sigma, \tau)$; b) no plano octaédrico (Ibañez, 2003).

Figura 4.2 - Curvas de deformação volumétrica para ensaios de cisalhamento cíclico de amplitude de deformação constante (modificado de Martin et. al., 1975).

Figura 4.3 - Curvas de deformação volumétrica incremental (adaptado de Martin et. al.,1975).

Figura 4.4 - Efeito da amplitude de deformação cisalhante cíclica e deformação volumétrica em relações de amplitudes de tensão $\mathrm{x}$ deformação drenadas, onde $1 \mathrm{psf}=47.9 \mathrm{~N} / \mathrm{m}^{2}$ (modificado de Martin,1975).

Figura 4.5 - Razão de incremento de poropressão em ensaios de cisalhamento simples cíclico (modificado de Seed et. al., 1975).

Figura 4.6 - Curva hiperbólica de tensão x deformação (adaptado de Finn et. al.,1977).

Figura 4.7 - (a) Ciclo do primeiro carregamento; (b) Mudança de volume com o tempo; (c) Detalhe da mudança de volume; (d) Modelo de carregamento geral. Modificado de Finn et. al. (1977).

Figura 4.8 - Curvas alternativas de deformação volumétrica dos dados das Figura 4.2 e Figura 4.3 (modificado de Byrne, 1991).

Figura 4.9 - Relação entre a razão de deformação volumétrica e o número de ciclos para areias secas (adaptado de Byrne,1991).

Figura 4.10 - Relação entre a deformação volumétrica e a deformação de cisalhamento para areias secas.

Dados de Silver \& Seed (1971) apud adaptado de Byrne (1991).

Figura 4.11 - Relação entre os níveis de tensões de cisalhamento e o número de ciclos para iniciar liquefação (modificado de Byrne,1991).

Figura 4.12 - Limite de escoamento do modelo UBCsand (adaptado de Byrne et al., 2004).

Figura 4.13 - Relação tensão - deformação hiperbólica

(modificado de Byrne et al., 2003). 
Figura 4.14 - Razão de tensões durante o descarregamento e

recarregamento (Park \& Byrne, 2004). 138

Figura 4.15 - Acoplamento do volume cisalhante (adaptado de Park, 2004). 140

Figura 5.1 - Condições de contorno aplicadas à estrutura no

programa FLAC (adaptado de Itasca, 2005).

Figura 5.2 - Erros introduzidos nas velocidades e deslocamentos pela

falta da correção da linha base no acelerograma.

(Modificado de Hudson (1979), apud de Carreño et al. (1999)).

Figura 5.3 - Variação da razão de amortecimento crítico normalizado com a frequência angular (Itasca, 2005).

Figura 5.4 - Curvas do fator de redução do módulo de

cisalhamento implementados no SHAKE e no FLAC

(modificado de Itasca, 2005).

Figura 5.5 - Curvas da razão do amortecimento crítico implementados

no SHAKE e no FLAC (adaptado de Itasca, 2005).

Figura 5.6 - Malha de campo livre (free-field) utilizada na avaliação

dinâmica no FLAC (adaptado de Itasca, 2005).

Figura 5.7 - Modelagem do contorno silencioso implementado

no FLAC (modificado de Itasca, 2005).

Figura 5.8 - Comparação para pequenas deformações (PD) e

grandes deformações (GD), do comportamento da poropressão durante

o sismo em diferentes pontos nodais da barragem do exemplo

No. 18 do manual do FLAC.

Figura 6.1 - Seção transversal da barragem de contenção de rejeitos.

Figura 6.2 - Parâmetros de resistência utilizados para o material de

enrocamento (adaptado de Leps,1970).

Figura 6.3 - Malha alternativa utilizada para avaliar a estrutura com a opção de grandes deformações.

Figura 6.4 - Configuração geométrica da barragem utilizada para a

avalição no programa FLAC.

Figura 6.5 - Variação da poropressão no rejeito em relação

à profundidade. Informação obtida com o CPT.

Figura 6.6 - Condição inicial de poropressão e condições de contorno 
simuladas no FLAC.

Figura 6.7 - Simulação da variação do módulo de cisalhamento em

função da tensão média efetiva no FLAC.

Figura 6.8- Modelagem do módulo de compressão volumétrica em

função do módulo de cisalhamento e do coeficiente de Poisson no FLAC.

Figura 6.9 - Valores de ângulo de atrito para a barragem.

Observa-se a variação na zona de enrocamento.

Figura 6.10 - Resposta estática da barragem em termos de tensões totais. $\quad 180$

Figura 6.11 - Valores de coesão na barragem.

Figura 6.12 - Superfície crítica obtida durante a avaliação da

estabilidade estática da barragem.

Figura 6.13 - Análise de estabilidade pseudo-estática da estrutura

efetuada no programa SLOPE/W.

Figura 6.14 - Valores de SPT do rejeito obtidos na investigação

geotécnica de campo.

Figura 6.15 - Função densidade de espectro de potência dos registros sísmicos utilizados para a geração do sismo artificial.

Figura 6.16 - Sismo gerado artificialmente utilizado na avaliação

dinâmica no FLAC.

Figura 6.17 - Potência do sismo avaliada na velocidade do registro sísmico.

Figura 6.18 - Comparação dos deslocamentos do registro sísmico

corrigido e não corrigido.

Figura 6.19 - História de acelerações para o sismo original e corrigido.

Figura 6.20 - Espectro de potência de alguns pontos nodais

localizados nos diferentes materiais que compõem a barragem.

Figura 6.21 - Localização das colunas de aferição na barragem de

contenção de rejeitos.

Figura 6.22 - Resultados da aferição da coluna 1 com relação à

tensão cisalhante máxima.

Figura 6.23 - Resultado da aferição com a coluna 1 considerando as acelerações máximas.

Figura 6.24 - Resultados da aferição da coluna 2 considerando a

tensão de cisalhamento máxima. 
Figura 6.25 - Resultados da aferição da coluna 2 com relação à aceleração máxima.

Figura 6.26 - Deslocamentos horizontais obtidos da avaliação dinâmica

utilizando o modelo de Byrne com o amortecimento histerético.

Figura 6.27 - Deslocamentos horizontais e verticais obtidos na avaliação

com o modelo Mohr-Coulomb. 194

Figura 6.28 - Deslocamentos obtidos na avaliação com o modelo de Byrne. 195

Figura 6.29 - Deslocamentos horizontais obtidos na avaliação dinâmica. 196

Figura 6.30 - Resposta dinâmica de deslocamentos verticais. 196

Figura 6.31 - Velocidades horizontais obtidas em diferentes pontos da barragem.

Figura 6.32 - Tensões cisalhantes obtidas no aterro como produto da avalição dinâmica. $\quad 198$

Figura 6.33 - Avaliação de liquefação na barragem. 198

Figura 6.34 - Razão de excesso de poropresões em pontos localizados a diferentes profundidades do rejeito.

Figura 6.35 - Poropressões geradas após a análise dinâmica.

Figura 6.36 - Fator de segurança não-drenado obtido na avaliação com o modelo de Mohr.

Figura 6.37 - Fator de segurança não-drenado obtido com o modelo de Byrne. 200 Figura 6.38 - Análise de estabilidade efetuada com o modelo de Mohr-Coulomb para o caso drenado.

Figura 6.39 - Fator de segurança drenado obtido na avaliação pós-sismica com o modelo de Byrne.

Figura 6.40 - Comparação da razão de excesso de poropressão no rejeito em função do fator de segurança contra liquefação com as curvas de Marcuson et al. (1990).

Figura 6.41 - Fator de segurança pós-sismo para valores de resistência residual reduzida utilizando o modelo de Byrne. 


\section{Lista de tabelas}

Tabela 2.1 - Terremotos no Brasil com magnitude superior a 5 entre 1922 e 2005

43

Tabela 2.2 - Coeficientes da lei de atenuação de Joyner \& Boore (1988) apud Kramer (1996).

53

Tabela 3.1 - Comparação das vantagens e desvantagens de vários ensaios de campo utilizados para a avaliação da resistência à liquefação (modificado de Youd et al., 2001).

Tabela 3.2 - Correções do SPT (modificado de Youd et al., 2001). 79

Tabela 3.3 - Correção por conteúdo de finos recomendado para a avaliação da resistência residual $\left(S_{r}\right)$ utilizando dados de SPT (Seed \& Harder, 1990). 91

Tabela 5.1 - Ajuste numérico para areias com as curvas de Seed \& Idriss (1970). Adaptado de Itasca (2005). 158

Tabela 6.1 - Propriedades dos Materiais utilizados no programa FLAC. 173

Tabela 6.2 - Parâmetros obtidos na avaliação da ameaça sísmica da estrutura. 184 


\section{Lista de Símbolos}

\section{Romanos}

\begin{tabular}{ll}
$A$ & Área \\
$A_{1}, A_{2}, A_{3}$ & Constantes \\
$B_{1}, B_{2}, B_{3}$ & Constantes \\
{$[C]$} & Matriz de amortecimento viscoso \\
$c$ & Coesão \\
$C_{B}$ & Fator de correção por diâmetro do furo de sondagem do ensaio \\
$C_{E}$ & SPT \\
$c_{n}$ & Fator de correção por energia do martelo do ensaio SPT \\
$C_{N}$ & Amplitude do enésimo harmônico das séries de Fourier \\
$C_{R}$ & Fator de correção por sobrecarga \\
$C_{S}$ & Fator de correção por comprimento da haste \\
$C_{v}$ & Fator de correção por amostradores com ou sem camisa \\
$C_{1}, C_{2}, C_{3}, C_{4}$, & Coeficiente de adensamento do solo \\
$C_{5}, C_{6}, C_{7}, C_{8}$, & Constantes \\
$C_{9}$ & \\
$d_{c}$ & Diâmetro do cone \\
$D_{r}$ & Densidade relativa \\
$d x / d y$ & Inclinação de curva \\
$\bar{E}_{r}$ & Módulo tangencial da curva de descarregamento \\
$e$ & unidimensional \\
$e_{S s}$ & Índice de vazios \\
$f_{c}$ & Índice de vazios na condição de estado permanente \\
$f_{m a x}$ & Frequência de esquina \\
$F_{M(m)}$ & Frequência de corte \\
$F S_{L}$ & Função densidade de probabilidade de magnitude m \\
$g$ & Fator de segurança contra a liquefação \\
& Aceleração gravitacional ou gravidade \\
& \\
\hline &
\end{tabular}




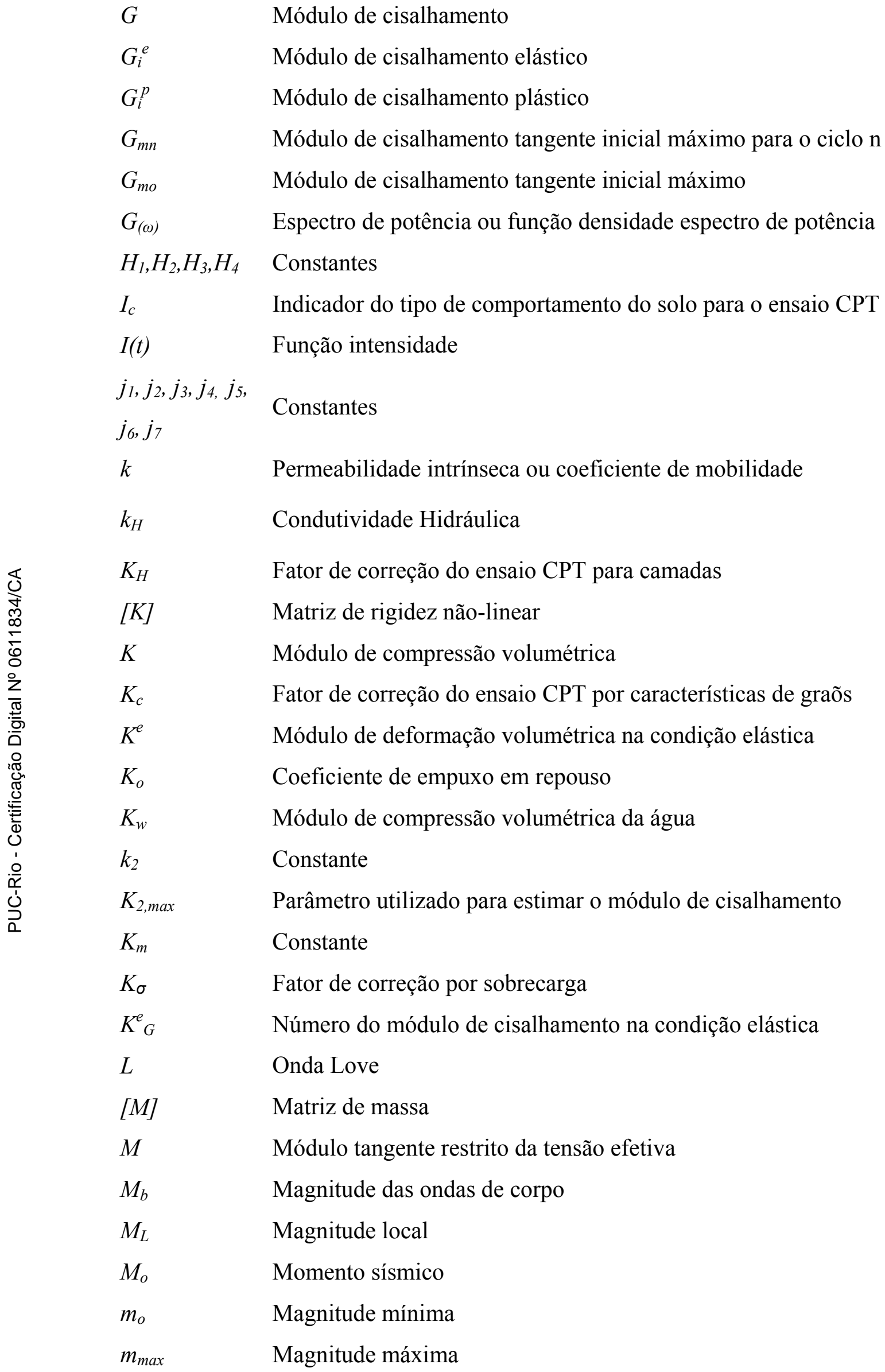




\begin{tabular}{|c|c|}
\hline$M_{S}$ & Magnitude de ondas superficiais \\
\hline$M_{w}$ & Magnitude de momento \\
\hline$n$ & Porosidade \\
\hline$N_{B C}$ & Contagem do número de golpes do ensaio BPT \\
\hline$N_{\text {corr }}$ & Função de porcentagem de finos \\
\hline$N_{e q}$ & Número de ciclos de tensões uniformes equivalente \\
\hline$N_{l}$ & Número de ciclos necessários para o inicia da liquefação \\
\hline$N_{m}$ & $\begin{array}{l}\text { Contagem do número de golpes do ensaio SPT medido no } \\
\text { campo }\end{array}$ \\
\hline$\left(N_{1}\right)_{60}$ & Contagem do número de golpes do ensaio SPT normalizado \\
\hline$\left(N_{1}\right)_{60-C S}$ & $\begin{array}{l}\text { Contagem do número de golpes do ensaio SPT normalizado } \\
\text { para areia limpa }\end{array}$ \\
\hline$p$ & Tensão total média \\
\hline$p^{\prime}$ & Tensão efetiva média \\
\hline$P$ & Onda primária \\
\hline$P_{a}$ & Pressão atmosférica \\
\hline$P_{(n)}$ & Função do modelo de Poisson \\
\hline$q$ & Tensão de desvio \\
\hline$q_{c}$ & Resistência à penetração da ponta do ensaio CPT \\
\hline$q_{C A}$ & $\begin{array}{l}\text { Resistência à penetração da ponta do ensaio CPT para camadas } \\
\text { rijas }\end{array}$ \\
\hline$q_{C B}$ & $\begin{array}{l}\text { Resistência à penetração da ponta do ensaio CPT para camadas } \\
\text { moles }\end{array}$ \\
\hline$q_{c 1 N}$ & Resistência de ponta do ensaio CPT normalizada \\
\hline$R$ & Onda Rayleigh \\
\hline$r_{d}$ & Coeficiente de redução de tensão \\
\hline$r_{u}$ & Razão de poropressão \\
\hline$S$ & Onda secundária ou de cisalhamento \\
\hline$s_{d}$ & Resistência ao cisalhamento médio do solo liquefeito \\
\hline$S H$ & Onda cisalhante horizontal \\
\hline$s_{s u}$ & Resistência ao cisalhamento no estado permanente \\
\hline$S V$ & Onda cisalhante vertical \\
\hline$s_{u}($ yield,mob) & Resistência não drenada mobilizada na condição de escoamento \\
\hline
\end{tabular}




$\begin{array}{ll}s_{u}(\text { critical }) & \text { Resistência não drenada crítica } \\ s_{u}(\text { LIQ }) & \text { Resistência ao cisalhamento na zona liqüefeita } \\ t & \text { Tempo } \\ T & \text { Período do sistema } \\ T_{d} & \text { Duração do sismo } \\ \ddot{u}_{g}(t) & \text { Aceleração do sismo } \\ v_{p} & \text { Velocidade da onda primária } \\ v_{s} & \text { Velocidade da onda de cisalhamento } \\ v_{s} & \text { Velocidade da onda de cisalhamento corrigida por sobrecarga } \\ W & \text { Peso } \\ x & \text { Deslocamento relativo } \\ \dot{x} & \text { Velocidade relativa } \\ \ddot{x} & \text { Aceleração relativa } \\ Y & \text { Parâmetro do movimento do terreno } \\ z & \text { Profundidade }\end{array}$




\section{Gregos}

$\alpha \quad$ Coeficiente de amortecimento local

$\Delta \quad$ Distância epicentral em graus

$\Delta \varepsilon_{v d} \quad$ Incremento de deformação volumétrica acumulada

$\Delta \varepsilon^{e}{ }_{v} \quad$ Deformação volumétrica incremental elástica

$\Delta \varepsilon^{p}{ }_{v} \quad$ Deformação volumétrica incremental plástica

$\Delta t \quad$ Incremento de tempo

$\Delta t_{p-s} \quad$ Diferença de chegada entre as onda $P$ e $S$

$\Delta u \quad$ Incremento de poropressão

$\varepsilon_{v} \quad$ Deformação volumétrica

$\varepsilon_{v d} \quad$ Deformação volumétrica acumulada

$\varepsilon_{v r} \quad$ Deformação volumétrica recuperável

$\varphi \quad$ Ângulo de atrito

$\varphi_{m o} \quad$ Ângulo de atrito mobilizado no plano horizontal

$\varphi_{c v} \quad$ Transformação de fase do ângulo de atrito para volume constante

$\varphi_{m l} \quad$ Ângulo de atrito mobilizado

$\gamma \quad$ Amplitude de deformação cisalhante

$\gamma^{*} \quad$ Deformação cisalhante plástica

$\gamma_{h} \quad$ Deformação hiperbólica

$\gamma^{p} \quad$ Deformação cisalhante na condição plástica

$\gamma_{r} \quad$ Amplitude de deformação cisalhante quando ocorre reversão de carregamento

$\gamma_{t} \quad$ Deformação limite

$\eta_{f} \quad$ Razão de tensão na ruptura

$\lambda \quad$ Constante de Lamé

$\lambda_{m} \quad$ Razão anual média de ultrapassagem da magnitude do sismo $\mathrm{m}$

$\theta \quad$ Ângulo de inclinação

$\rho \quad$ Massa específica

$\rho_{\omega} \quad$ Massa específica da água

$\mu \quad$ Constante de Lamé

$v_{p} \quad$ Velocidade da onda $P$

$v_{s} \quad$ Velocidade da onda $S$ 


$\begin{array}{ll}\sigma & \text { Tensão média total } \\ \sigma^{\prime} & \text { Tensão média efetiva } \\ \sigma_{m}^{\prime} & \text { Tensão efetiva média normal } \\ \sigma_{v o}^{\prime} & \text { Tensão efetiva vertical inicial } \\ \tau_{l} & \text { Tensão de cisalhamento máxima } \\ \tau_{d} & \text { Tensão cisalhante requerida para manter o equilíbrio estático } \\ \tau_{h v} & \text { Amplitude de tensão de cisalhamento cíclica } \\ \tau_{m o} & \text { Tensão cisalhante máxima } \\ \tau_{m n} & \text { Tensão cisalhante máxima para o ciclo n } \\ \tau_{r} & \text { Tensão cisalhante quando ocorre reversão de carregamento } \\ v & \text { Coeficiente de Poisson } \\ \psi & \text { Parâmetro de estado } \\ \psi_{l} & \quad \text { Ângulo de dilatância } \\ \omega & \text { Frequência natural do sistema } \\ \xi & \text { Amortecimento }\end{array}$




\section{Lista de Abreviaturas}

BPT Ensaio de penetração Becker

CPT Ensaio de penetração de cone

CSR Razão de tensão cíclica ou razão de tensão cisalhante cíclica

CRR Razão de resistência cíclica ou razão de resistência ao cisalhamento cíclica

DBE Sismo base de projeto

DLLs Livrarias dinâmicas

DSHA Análise de ameaça sísmica determinística

ELM Método linear equivalente

EMS-98 Escala macrosísmica europeia de intensidade de sismo

ER Razão de energia

FC Conteúdo de finos

FDEP Função de densidade de espectro de potência

FFT Transformada rápida de Fourier

FLAC Finite Lagrangian Analysis of Continua.

GIIC Graphical Interface for Itasca Codes

JMA Escala de intensidade de sismo da agencia meteorológica japonesa

MCE Sismo máximo a ser considerado

MDE Sismo máximo de projeto

MEF Método de elementos finitos

MMI Escala de Intensidade de sismo de Mercalli modificada

MSF Fator de correção do ensaio de penetração padrão pela magnitude do sismo

MSK Escala de intensidade de sismo Medvedev-Sponnheuer-Karnik

NCEER National Center Earthquake Engineering Research

OBE Sismo base de operação

PHA Aceleração horizontal de pico

PHV Velocidade horizontal de pico

PSHA Análise de ameaça sísmica probabilística 
RF Escala de intensidade de sismo Rosel-Forel

SDOF Sistema de um grau de libertade

SPT Ensaio de penetração padrão

SSE Sismo de desligamento seguro

SSL Linha do estado permanente 